\title{
Thermoelectric Properties of p-Type PbSe Nanowires
}

\author{
Wenjie Liang ${ }^{1,3}$, Oded Rabin ${ }^{1,4}$, Allon I. Hochbaum ${ }^{1}$, Melissa Fardy ${ }^{1}$, Minjuan Zhang ${ }^{2}$, and Peidong Yang ${ }^{1}(\varangle)$ \\ ${ }^{1}$ Department of Chemistry, University of California, Berkeley, CA 94720, USA \\ ${ }^{2}$ Materials Research Department, Toyota Technical Center, Toyota Motor Engineering \& Manufacturing North America (TEMA) Inc., \\ 1555 Woodridge Ave., Ann Arbor, MI 48105, USA \\ ${ }^{3}$ Current Address: Institute of Physics, Chinese Academy of Sciences, Beijing 100080, China \\ ${ }^{4}$ Current Address: Department of Materials Science and Engineering and the Institute for Research in Electronics and Applied Physics, \\ University of Maryland, College Park, MD 20742, USA \\ Received: 12 February 2009 / Revised: 11 March 2009 / Accepted: 11 March 2009 \\ CTsinghua University Press and Springer-Verlag 2009. This article is published with open access at Springerlink.com
}

\begin{abstract}
The thermoelectric properties of individual solution-phase synthesized p-type PbSe nanowires have been examined. The nanowires showed near degenerately doped charge carrier concentrations. Compared to the bulk, the PbSe nanowires exhibited a similar Seebeck coefficient and a significant reduction in thermal conductivity in the temperature range $20 \mathrm{~K}$ to $300 \mathrm{~K}$. Thermal annealing of the PbSe nanowires allowed their thermoelectric properties to be controllably tuned by increasing their carrier concentration or hole mobility. After optimal annealing, single PbSe nanowires exhibited a thermoelectric figure of merit (ZT) of 0.12 at room temperature.
\end{abstract}

\section{KEYWORDS}

Nanowire, thermoelectrics, thermopower, thermal conductivity, lead chalcogenide

Solid state thermoelectric devices can convert thermal gradients into electrical power and have attracted increased interest for energy applications [1]. High performance, reliability, portability, and low cost are among the key characteristics that make these solid state devices attractive for practical applications. Efficient thermal to electrical power conversion requires low thermal conductivity to sustain a good temperature gradient and high charge carrier mobility to generate electrical power. The device performance is characterized by the thermoelectric figure of merit, $\mathrm{ZT}=S^{2} \sigma \mathrm{T} / \mathrm{K}$, where $S$ is the thermopower (Seebeck coefficient), $\sigma$ is the electrical conductivity, $T$ is the absolute temperature, and $K$ is the thermal conductivity from both lattice and charge carrier contributions. For a solid state thermoelectric module to compete on the commercial market, a value of $\mathrm{ZT}$ of three or greater is considered necessary. Due to the interrelated nature of $S, \sigma$, and $K$, traditional bulk materials have not achieved a value of ZT greater than unity [2]. Recently, theoretical predictions [3, 4] and experimental measurements [5-11] indicated that

Address correspondence to p_yang@berkeley.edu 
higher ZT values could be obtained in nanomaterials by tailoring the band structure and density of states (DOS) in low-dimensional systems and enhancing phonon boundary scattering.

Bulk lead chalcogenides have traditionally been used as thermoelectric materials due to their low thermal conductivity $\left(\sim 2 \mathrm{~W} \cdot \mathrm{m}^{-1} \cdot \mathrm{K}^{-1}\right.$ for PbSe at room temperature for example). Dimensional reduction could further boost their thermoelectric properties. Recently, Wang et al. [9] showed that thermopower increased monotonically in PbSe quantum dot films with decreasing quantum dot size, and in 2002, Harman et al. [5] reported $\mathrm{PbSeTe} / \mathrm{PbTe}$ quantum dot superlattices with $\mathrm{ZT} \approx 2$. Compared with nanoparticle films, one-dimensional nanowires are characterized by better electrical properties. The solution-phase synthesis of PbSe nanowires represents a low-cost and potentially scalable process to generate large amounts of thermoelectric nanomaterials. Here we report a comprehensive study of the thermoelectric properties of solution-phase synthesized PbSe nanowires, as well as property enhancement after annealing.

The synthesis of jagged PbSe nanowires was similar to that reported by Cho et al. [12]. Briefly, 0.76 $\mathrm{g}$ of lead acetate trihydrate and $2 \mathrm{~mL}$ of oleic acid in $10 \mathrm{~mL}$ of diphenyl ether were heated at $150{ }^{\circ} \mathrm{C}$ for 30 min to remove any water from the solution and form lead oleate. After cooling the lead oleate solution to $60{ }^{\circ} \mathrm{C}, 4 \mathrm{~mL}$ of $0.16 \mathrm{~mol} / \mathrm{L}$ trioctylphosphine selenide (TOPSe) in trioctylphosphine (TOP) were injected into the lead oleate solution and the combined solution was then injected into $15 \mathrm{~mL}$ of dry diphenyl ether at $250{ }^{\circ} \mathrm{C}$. After heating for $2 \mathrm{~min}$ the reaction was quenched in a water bath. The entire procedure was performed under inert gas. The product was washed with hexanes and centrifuged at $3000 \mathrm{rpm}$ for 30 min and then stored in either hexanes or chloroform. The nanowires remained stable in solution for a period of over a year. The resulting PbSe nanowires exhibited jagged surfaces due to oriented attachment of PbSe nanoparticles during the nanowire growth. Figure 1(a) shows a transmission electron microscopy (TEM) image of the as-synthesized nanowires. The diameters of the nanowires ranged from $50 \mathrm{~nm}$ to 100 $\mathrm{nm}$, and the diameter could be tuned by altering the heating time after precursor injection. The visibly

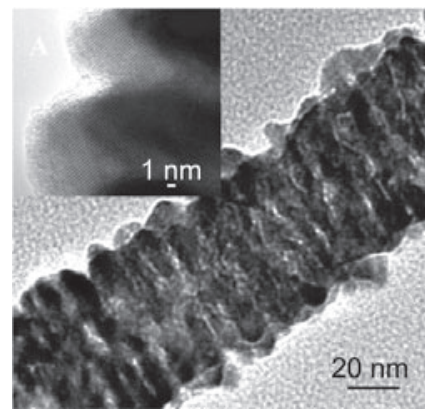

(a)

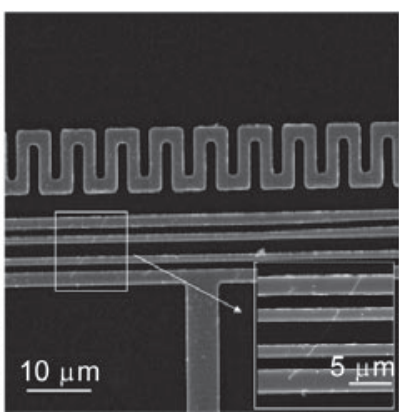

(b)
Figure 1 (a) TEM image of solution-phase synthesized PbSe nanowire. Inset: high-resolution TEM image of a PbSe nanowire. (b) SEM image of a device used for individual nanowire thermoelectric measurements. The device was fabricated on a $\mathrm{Si} / \mathrm{SiO}_{2}$ chip with a coil electrode designed to generate a temperature gradient. Inset: SEM image of the device with a single PbSe nanowire contacted by four 1 $\mathrm{nm} / 100 \mathrm{~nm} / 30 \mathrm{~nm}$ Ti/Pd/Au electrodes

rough surfaces of the PbSe nanowires suggest that they may have a reduced thermal conductivity due to phonon surface scattering $[7,13,14]$. While it initially appeared that the nanowires are composed of chains of nanoparticle domains, as can be seen in the high-resolution TEM image in the inset, the nanowire was single-crystalline. The single-crystalline nature of these nanowires could provide a major advantage over nanoparticle superlattice films where the electrical conductivity is significantly limited by charge scattering, tunneling, and hopping.

Electrical, thermopower, and thermal conductivity studies were all based on single nanowire measurements, allowing the intrinsic properties of the nanowire to be clearly distinguished from ensemble effects. Prior to device fabrication, the asmade PbSe nanowires were washed with ethanol, followed by centrifugation and redispersal in chloroform. The devices used for thermopower and electrical measurements were constructed in the same way except the thermopower devices were fabricated on Pyrex glass and the electrical devices were fabricated on either Pyrex or Si wafers with a $600 \mathrm{~nm}$ coating of thermal oxide. The devices were made by spin-casting the nanowire dispersion onto the desired wafer and using standard photolithography procedures to define electrodes and a heater. The metal contacts were $1 \mathrm{~nm} / 100 \mathrm{~nm} / 30 \mathrm{~nm} \mathrm{Ti} / \mathrm{Pd} /$ $\mathrm{Au}$ deposited with an e-beam evaporator. We note here that these PbSe nanowires were very fragile, so 
extra care was taken to avoid mechanical stress and thermal shock during the device fabrication. All devices were stored in a desiccator under vacuum and measurements were performed in air. The electrical properties of the nanowires slowly degraded if exposed to air and after several days the nanowires become insulating. When kept in a desiccator they could be preserved for months.

Figure 1(b) shows a scanning electron microscopy (SEM) image of a device with a single PbSe nanowire bridging four electrodes. A heater was positioned next to the electrodes to generate a temperature gradient in the direction normal to that of the electrodes $[7,8]$. Any pair of electrodes could be used to measure the electrical conductance and thermal voltage generated in the PbSe nanowire when heated. The middle two electrodes were each attached to four electrodes so the change in their resistance as a function of temperature could be recorded and calibrated, allowing them to serve as temperature sensors for thermopower measurements.

Four-point probe measurements were used to study the electrical properties of single $\mathrm{PbSe}$ nanowires. A voltage was applied between the outer two electrodes using a National Instruments 6052E multifunction data acquisition (DAQ) card while the drain current $(I)$ on the outer electrodes and voltage $(V)$ between the inner two electrodes were monitored using a DL 1211 current preamplifier and Stanford Research Systems SR560 voltage preamplifier, respectively. The field-effect properties of single PbSe nanowires were measured by applying a gate voltage $\left(V_{\mathrm{g}}\right)$ to the device. Current passing through the nanowire evolved linearly with voltage, suggesting that the $\mathrm{Ti} / \mathrm{Pd} / \mathrm{Au}$ formed ohmic contacts to the PbSe nanowire. Two-point probe and fourpoint probe resistance measurements confirmed that the contact resistance was indeed negligible. The asmade PbSe nanowires had resistivities ranging from 0.1 to $1 \Omega \cdot \mathrm{cm}$.

$I-V$ characteristics of PbSe nanowire devices were studied under different gate voltages [15]. Plots of the conductance $(G)$ vs $V_{\mathrm{g}}$ of single nanowire devices show that increasingly negative values of $V_{\mathrm{g}}$ led to more conductive PbSe nanowires. This trend indicates that the PbSe nanowire has hole-dominated charge transport and that the nanowire is a p-type semiconductor. A large proportion of the measured PbSe nanowire devices with ohmic contacts showed no change in conductance with varying gate voltage, suggesting that they were degenerately doped with holes. A carrier concentration of $10^{19} \mathrm{~cm}^{-3}$ and higher is ideal for semiconducting thermoelectrical materials. Hence, our as-made PbSe nanowires exhibit a suitable carrier concentration without the need to introduce other dopant species.

The temperature dependent four-point probe resistivity is shown in Fig. 2(a). As the temperature decreased from $300 \mathrm{~K}$, resistivity initially dropped sharply and then increased more slowly. This temperature dependence was reproducible, although the amplitude of the change in resistivity varied from device to device. The metallic-type temperature dependence near room temperature is consistent with the fact that the PbSe nanowires are heavily doped and charge carrier scattering predominates. At lower temperatures ( $200 \mathrm{~K}$ to $100 \mathrm{~K})$, on the other hand, thermal excitation of charge carriers dominates. From the temperature dependent resistivity plot, the calculated thermal excitation energy was $10 \mathrm{meV}$. When temperature dropped below $100 \mathrm{~K}$, the fourpoint probe $I-V$ curves became non-linear, suggesting that an energy dependent scattering mechanism operates inside the nanowires.

Devices for thermopower measurement were fabricated on Pyrex glass substrates in order to achieve larger temperature gradients at a given heating power. A Keithley 236 source-measurement unit was used as a current source to generate heat in the heater, while the thermal voltage between the attached electrodes on the hot and cold ends of a single PbSe nanowire was recorded using a Keithley 2182 nanovoltmeter. The sign convention is such that a positive voltage indicates that hole carriers are responsible for thermal transport. Figure 2(b) shows a plot of measured thermal voltage from a PbSe nanowire as a function of heating current. Positive thermal voltages were generated and the voltages evolved in a parabolic fashion with heating current. A heating current of $8 \mathrm{~mA}$ produced a thermal voltage of $920 \mu \mathrm{V}$ across the nanowire. During the heating process the change in resistance of the two 
thermal sensors was recorded using two lock-in amplifiers. The temperatures of the electrodes were calibrated using temperature coefficient of resistance (TCR) measurements. The thermopower is given by $S=V_{\mathrm{s}} / \Delta T$, where $V_{\mathrm{s}}$ is the thermal voltage and $\Delta T$ is the temperature difference across the nanowire. More than 10 samples were measured and their thermopower ranged from $223 \mu \mathrm{V} / \mathrm{K}$ to $445 \mu \mathrm{V} / \mathrm{K}$ with an average of $339 \mu \mathrm{V} / \mathrm{K}$. These values are comparable to that of bulk PbSe [16] with comparable charge carrier concentrations.

To measure the thermal conductivity of individual PbSe nanowires, they were drop-cast onto prefabricated devices [17-19]. The devices consisted of suspended $\mathrm{SiN}_{x}$ heating and sensing pads, each with a Pt heating coil and four Pt contact lines. Once an individual nanowire was found bridging the heating and sensing pads, it was bonded with $\mathrm{Pt}$ to create a better thermal interface (Fig. 2(c) inset). To measure the thermal conductivity of a suspended nanowire, the temperature of one pad was increased while the temperature change of the opposite pad was recorded. An increase in temperature of the sensing pad was due to heat transfer from the heating pad through the PbSe nanowire. The nanowire dimensions were estimated from SEM images and the thermal conductivity was calculated using methods described in previous reports [17-19]. The thermal conductivity of an individual $\mathrm{PbSe}$ nanowire was $0.8 \mathrm{~W} \cdot \mathrm{m}^{-1} \cdot \mathrm{K}^{-1}$ at $300 \mathrm{~K}$, and below $100 \mathrm{~K}$ the thermal conductivity dropped drastically, possibly due to phonon boundary scattering.

At $300 \mathrm{~K}$, a ZT value of $\sim 0.04$ was estimated for single, as-made PbSe nanowires by combining the results of electrical, thermopower, and thermal conductivity measurements. One reason for this low value of $\mathrm{ZT}$ is that the charge carrier mobility of the nanowires is still two orders of magnitude lower than that achieved in the bulk $[15,20]$. This reduction in mobility could be a result of surface trap states and point defects, which can serve as scattering centers.

Thermal annealing has been shown to reduce nanowire defect density [21] and could possibly help improve the thermoelectric properties of the PbSe nanowire. Pyrex chips holding PbSe nanowire devices were therefore annealed at $180{ }^{\circ} \mathrm{C}$ to $250^{\circ} \mathrm{C}$ in a tube

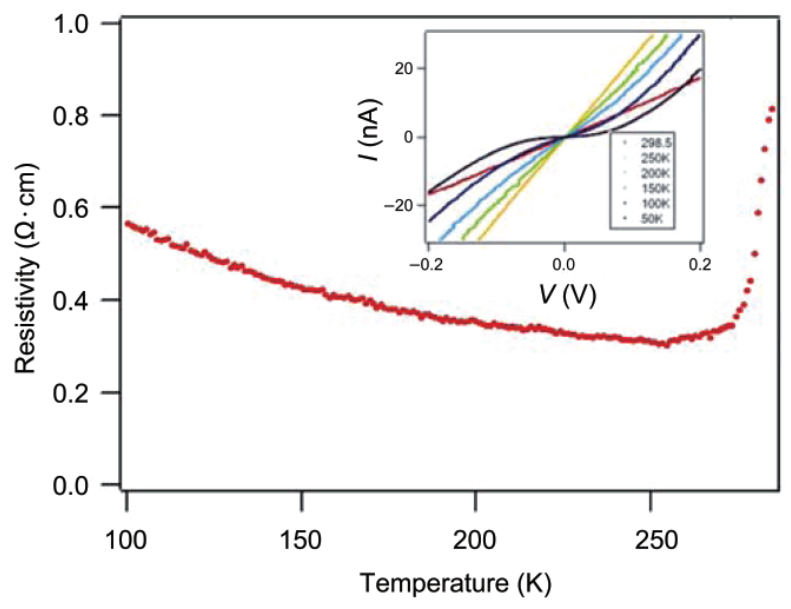

(a)

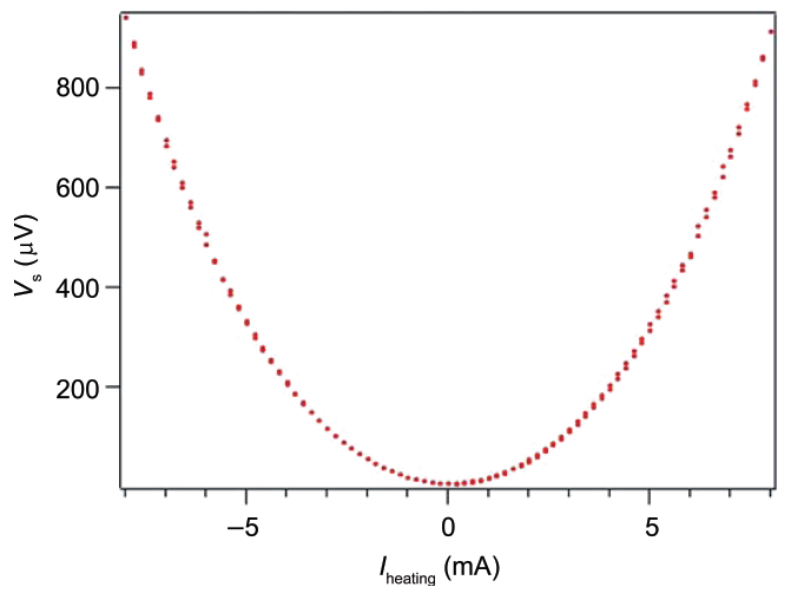

(b)

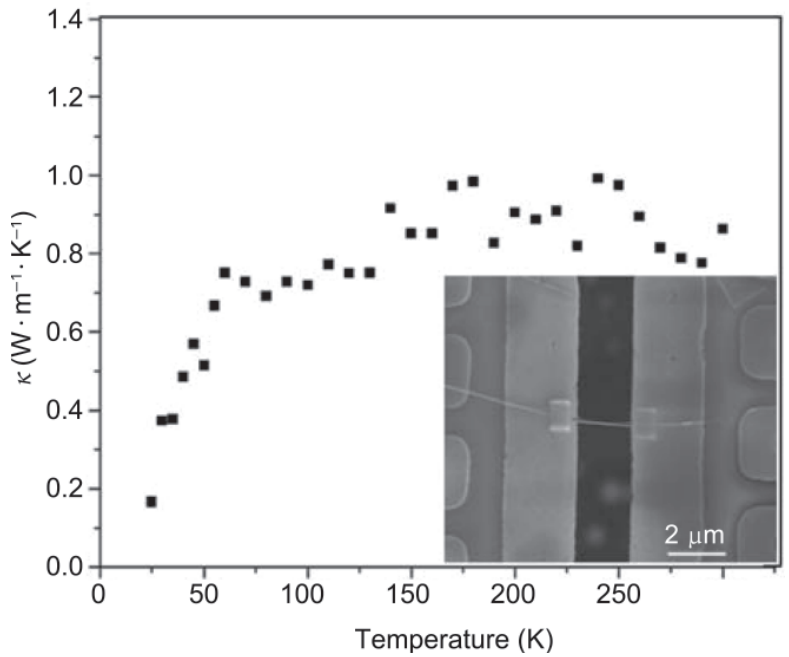

(c)

Figure 2 Themoelectrical measurements of single as-made PbSe nanowires. (a) Temperature dependent resistivity measurement. Inset: four-point probe measurement of $I-V$ at different temperatures. (b) Thermal voltage measured across a single PbSe nanowire (device shown in Fig. 1(b)) as a function of heating current. (c) Thermal conductivity of a single PbSe nanowire as a function of the temperature. Inset: SEM image of the measured device 
furnace under a nitrogen pressure of 1 Torr. Electrical properties were measured before and after annealing under different conditions. Figure 3(a) (inset) shows $I-V$ curves for a PbSe nanowire annealed at $200{ }^{\circ} \mathrm{C}$ for various durations. The color-coded $I-V$ curves represent the nanowire after annealing times of 0 , $2,3,4.5$, and $5 \mathrm{~h}$. A significant increase in electrical conductance was observed during the annealing process. After $5 \mathrm{~h}$, the nanowire conductance increased to 14 times its value prior to annealing. Figure 3(a) summarizes the enhancement in electrical conductance under different annealing conditions. Essentially, by changing the annealing temperature and time, the electrical conductance could be tuned by up to three orders of magnitude. Thus, the thermoelectric properties of individual PbSe nanowires could be explored as a function of resistivity from $0.4 \Omega \cdot \mathrm{cm}$ to $4 \times 10^{-4} \Omega \cdot \mathrm{cm}$. TEM studies showed no noticeable change in crystallinity or morphology of the nanowire due to the annealing process.

The thermopower of annealed PbSe nanowire devices as a function of nanowire resistivity is summarized in Fig. 3(b). The Seebeck coefficient was positive for all samples, indicating that holes were still responsible for charge transport in the annealed nanowires. The Seebeck coefficient decreased with decreasing resistivity, strongly suggesting that the hole concentration increased with annealing time and temperature. This increase in hole concentration could result from an increase in $\mathrm{Pb}$ vacancies during the annealing process.

The power factor $\left(S^{2} \sigma\right)$ and $\mathrm{ZT}$ at $300 \mathrm{~K}$ as a function of resistivity are shown in Figs. 3(c) and 3(d), respectively. As the resistivity decreased, the power factor and value of ZT increased until reaching a peak around $0.04 \Omega \cdot \mathrm{cm}$. The highest room temperature

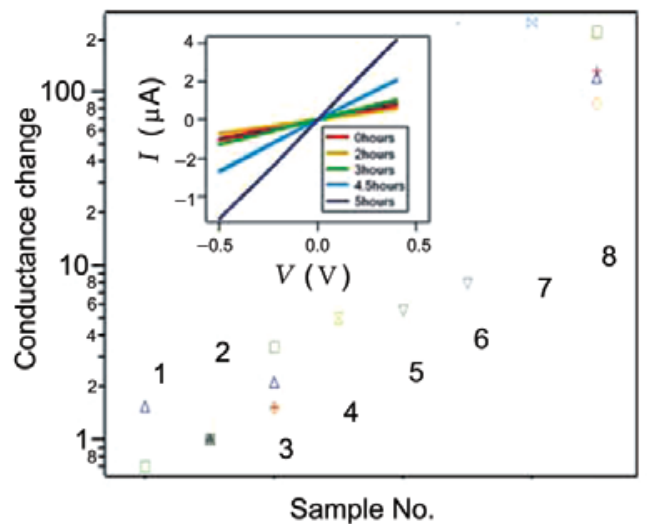

(a)

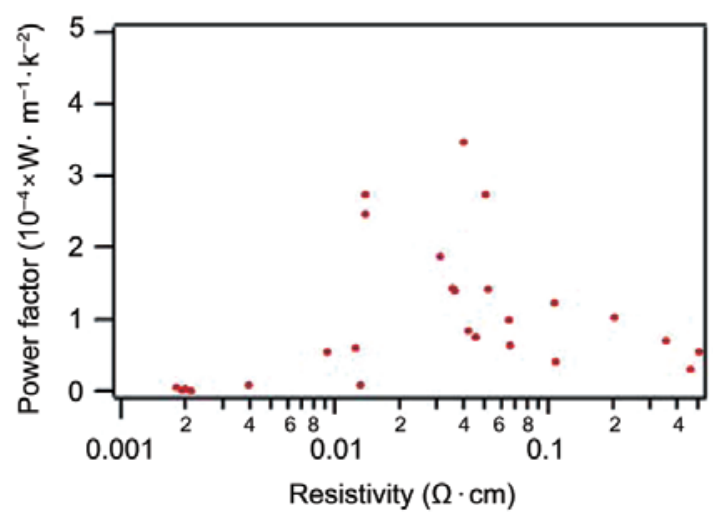

(c)

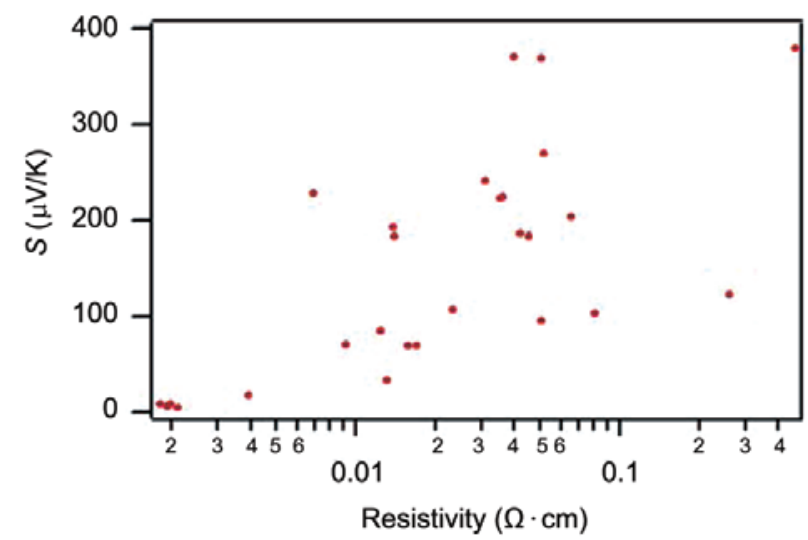

(b)

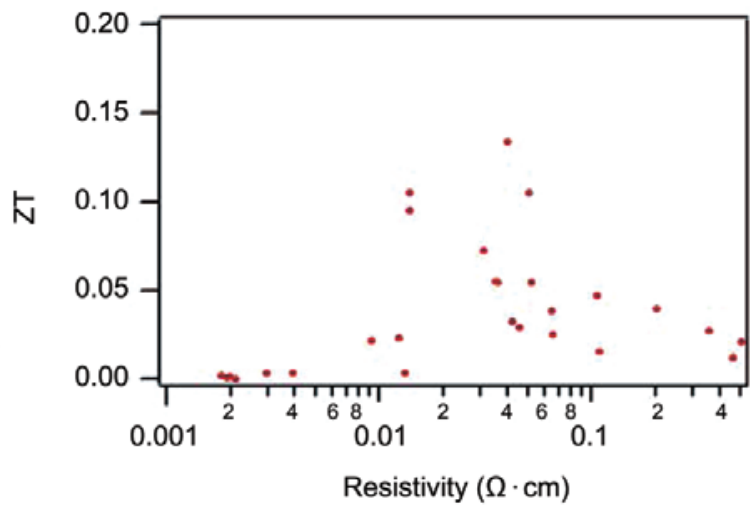

(d)

Figure 3 Thermoelectrical measurements of the PbSe nanowire devices after thermal annealing. (a) Ratio of conductivity change under the following annealing conditions. Sample \#1. before annealing; \#2. $180{ }^{\circ} \mathrm{C}, 2 \mathrm{~h}$; \#3. $180{ }^{\circ} \mathrm{C}, 10 \mathrm{~h} ; \# 4.200{ }^{\circ} \mathrm{C}, 0.5 \mathrm{~h}$; $\# 5.200{ }^{\circ} \mathrm{C}, 1 \mathrm{~h} ; \# 6.200^{\circ} \mathrm{C}, 4 \mathrm{~h} ; \# 7.200^{\circ} \mathrm{C}, 7 \mathrm{~h}$; \#8. $250^{\circ} \mathrm{C}, 7 \mathrm{~h}$. Inset: I-V curves of a single PbSe nanowire annealed at $200{ }^{\circ} \mathrm{C}$ for different durations. (b) Seebeck coefficient of annealed nanowires as a function of their resistivity. (c) Power factor of annealed nanowires as a function of their resistivity. (d) ZT of annealed nanowires as a function of their resistivity at $300 \mathrm{~K}$ 
power factor and value of $\mathrm{ZT}$ observed in the measured devices were $3.5 \times 10^{-4} \mathrm{~W} \cdot \mathrm{m}^{-1} \cdot \mathrm{K}^{-2}$ and 0.12 , respectively. The maximum value of $\mathrm{ZT}$ achived was three times that of the as-made nanowires.

To summarize, the thermoelectrical properties of solution-phase synthesized PbSe nanowires have been studied at the single nanowire level. Both fieldeffect transport and thermopower measurements showed that the as-made nanowires were p-type semiconductors with high carrier concentration. At $300 \mathrm{~K}$ the thermal conductivity of PbSe nanowires was $0.8 \mathrm{~W} \cdot \mathrm{m}^{-1} \cdot \mathrm{K}^{-1}$, which is two-fold smaller than that of the bulk material [22]. Controlled thermal annealing proved to be a feasible method for tuning the thermoelectric properties of the PbSe nanowires.

\section{References}

[1] Majumdar, A. Thermoelectricity in semiconductor nanostructures. Science 2004, 303, 777-778.

[2] Goldsmid, H. Thermoelectric Refrigeration; Plenum Press: New York, 1964.

[3] Hicks, L. D.; Dresselhaus, M. S. Effect of quantum-well structures on the thermoelectric figure of merit. Phys. Rev. B 1993, 47, 12727-12731.

[4] Hicks, L. D.; Dresselhaus, M. S. Thermoelectric figure of merit of a one-dimensional conductor. Phys. Rev. B 1993, 47, 16631-16634.

[5] Harman, T. C.; Taylor, P. J.; Walsh, M. P.; LaForge, B. E. Quantum dot superlattice thermoelectric materials and devices. Science 2002, 297, 2229-2232.

[6] Venkatasubramanian, R.; Siivola, E.; Colpitts, T.; O'Quinn, B. Thin-film thermoelectric devices with high roomtemperature figures of merit. Nature 2001, 413, 507602.

[7] Hochbaum, A. I.; Chen, R. K.; Delgado, R. D.; Liang, W. J.; Garnet, E. C.; Najarian, M.; Majumdar, A.; Yang, P. D. Enhanced thermoelectric performance of rough silicon nanowires. Nature 2008, 451, 163-167.

[8] Boukai, A.; Bunimovich, Y.; Tahir-Kheli, J.; Yu, J. K.; Goddard, W. A.; Heath, J. R. Silicon nanowires as efficient thermoelectric materials. Nature 2008, 451, 167-169.

[9] Wang, R. Y.; Feser, J. P.; Lee, J. S.; Talapin D. V.; Segalman, R.; Majumdar, A. Enhanced thermopower in PbSe nanocrystal quantum dot superlattices. Nano Lett. 2008, 8, 2283-2288.
[10] Hsu, K.F.; Loo, S. Guo, F.; Chen, W.; Dyck, J. S.; Uher, C.; Hogan, T.; Polychroniadis, E. K.; Kanatzidis, M. G. Cubic $\mathrm{AgPb}_{m} \mathrm{SbTe}_{2+m}$ : Bulk thermoelectric materials with high figure of merit. Science 2004, 303, 818-821.

[11] Poudel, B.; Hao, Q.; Ma, Y.; Lan, Y. C.; Minnich, A.; Yu, B.; Yan, X.; Wang, D. Z.; Muto, A.; Vashaee, D; Chen, X. Y.; Liu, J. M.; Dresselhaus, M. S; Chen, G.; Ren, Z. Highthermoelectric performance of nanostructured bismuth antimony telluride bulk alloys. Science, 2008, 320, 634638.

[12] Cho, K.; Talapin, D. V.; Gaschler, W.; Murray, C. B. Designing PbSe nanowires and nanorings through oriented attachment of nanoparticles. J. Am. Chem. Soc. 2005, 127, 7140-7147.

[13] Zou, J.; Balandin, A. Phonon heat conduction in a semiconductor nanowire. J. Appl. Phys. 2001, 89, 29322938.

[14] Moore, A. L.; Saha, S. K.; Prasher, R. S.; Li, S. Phonon backscattering and thermal conductivity suppression in sawtooth nanowires. Appl. Phys. Lett. 2008, 93, 083112.

[15] Liang, W. J.; Hochbaum, A. I.; Fardy, M.; Rabin, O.; Zhang, M. J.; Yang, P. D. Field-effect modulation of Seebeck coefficient in single PbSe nanowires. Nano Lett. 2009, 9, 1689-1693.

[16] Abrams, H.; Tauber, R. N. Thermoelectric power of singlecrystal p-type PbSe. J. Appl. Phys. 1969, 40, 3868-3870.

[17] Li, D.; Wu, Y.; Kim, P.; Shi, L.; Yang, P. D.; Majumdar, A. Thermal conductivity of individual silicon nanowires. Appl. Phys. Lett. 2003, 83, 2934-2936.

[18] Fardy, M. Hochbaum, A.; Goldberger, J.; Zhang, M. M.; Yang, P. D. Synthesis and thermoelectrical characterization of lead chalcogenide nanowires. Adv. Mater. 2007, 19, 3047-3051.

[19] Shi, L.; Li, D. Y.; Yu, C. H.; Jang, W. Y.; Kim. D.; Yao, Z.; Kim, P.; Majumdar, A. Measuring thermal and thermoelectric properties of one-dimensional nanostructures using a microfabricated device. J. Heat Transf. 2003, 125, 881888.

[20] Allgaier, R.; Scanlon, W. Moblity of electrons and holes in $\mathrm{PbS}, \mathrm{PbSe}$, and $\mathrm{PbTe}$ between room temperature and 4.2-degrees-K. Phys. Rev. 1958, 111, 1029-1037.

[21] Tang, Y. H.; Zheng, Y. F.; Lee, C. S.; Lee, S. T. A simple route to annihilate defects in silicon nanowires. Chem. Phys. Lett. 2000, 328, 346-349.

[22] Gray, D. E. American Institute of Physics Handbook, 3rd ed; McGraw-Hill: New York, 1972. 